\title{
Compact Multiantenna
}

\section{Rudant, C. Delaveaud, and P. Ciais}

CEA-Leti, Minatec Campus, 17 Rue des Martyrs, 38054 Grenoble Cedex 9, France

Correspondence should be addressed to L. Rudant, lionel.rudant@cea.fr

Received 30 July 2011; Accepted 30 October 2011

Academic Editor: Li Yang

Copyright (C) 2012 L. Rudant et al. This is an open access article distributed under the Creative Commons Attribution License, which permits unrestricted use, distribution, and reproduction in any medium, provided the original work is properly cited.

Planar inverted-f antenna (PIFA) and notch antenna are combined within a compact 2-port MIMO antenna. Electrical and magnetic duality of the two antennas avoids a critical coupling and best performances can be expected for multiple-input multiple-output (MIMO) communication. When excitation of notch antenna is optimized properly, the notch length can be short enough so that the two antennas can be colocated in a single compact volume. This compact multiantenna design is suitable for integration in MIMO handheld terminals. A prototype for broadband network application in 3.4-3.8 GHz frequency band has been characterized in anechoic chamber.

\section{Introduction}

Modern wireless networks have to meet the increasing needs in terms of throughput and reliability. Broadband communication and multiple-input multiple-output (MIMO) techniques are promising solutions. If multiantenna are widely developed and used at base station and access point side, a key challenge is without a doubt the design of compact and wideband MIMO antennas for small terminals.

Due to small size of modern terminals, multiantenna has to be integrated in restricted volume with respect to the wavelength. When space between antennas is too reduced, diversity must be achieved with an optimization of radiation, that is, pattern shape and electromagnetic field polarization. In addition to classical antenna miniaturization effects on radiation efficiency and bandwidth, multiantenna performances are impacted by high coupling when antennas are very close. In this paper, two radiating elements are colocated in a single electrically small volume with dual polarization and pattern diversity. Bandwidth, radiation efficiency, low coupling, and uncorrelated radiation properties have been optimized despite closeness.

Planar inverted-f antenna (PIFA) and notch antenna are common radiating elements to be integrated at terminal because of small size. Different combinations of PIFA and notch have been reported in [1] for compact MIMO antenna design. When the two elements are combined side by side, a slit can cut between them in the ground plane to avoid high coupling [2]. In [3], a partial overlap of PIFA and notch has been presented.

Earlier in [4], PIFA and notch have been brought together in colocated multiantenna system. In this paper, PIFA and notch are combined with complete overlap and diversity can be integrated in a single antenna volume. A prototype for broadband MIMO communication is characterized in anechoic chamber.

\section{Close Combination of PIFA and Notch}

Polarization diversity is commonly performed with linearly polarized radiating elements which are oriented orthogonally. Planar inverted-f antenna (PIFA) is electric-like radiating element and notch antenna is considered as magneticlike radiating slot [4]. The combination presented in Figure 1 enables achieving complementary electric/magnetic diversity with a compact multiantenna because both elements are quarter-wavelength resonators. One other advantage is that the two combined elements have collinear resonant lengths and multiantenna can fit a thin volume in the terminal.

When notch is fed by coupling with a printed micro-strip line, impedance matching is reached by joint optimization of two parameters: $a$ is relative position of the strip along the notch; $p$ is serial stub length after the notch. In Figure 2, a notch antenna is optimized at $2.5 \mathrm{GHz}$ frequency band with 


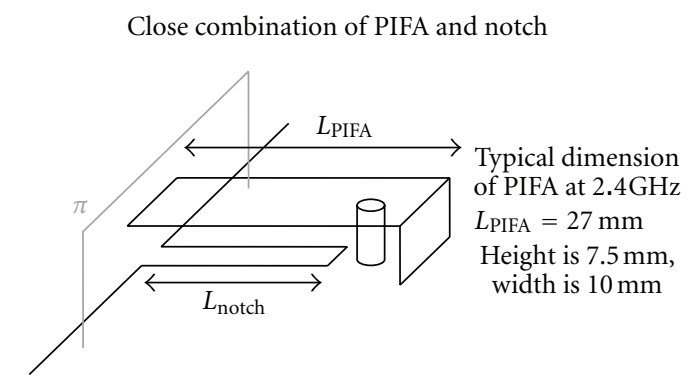

FIgURE 1: Close combination of PIFA and notch with complete overlap.
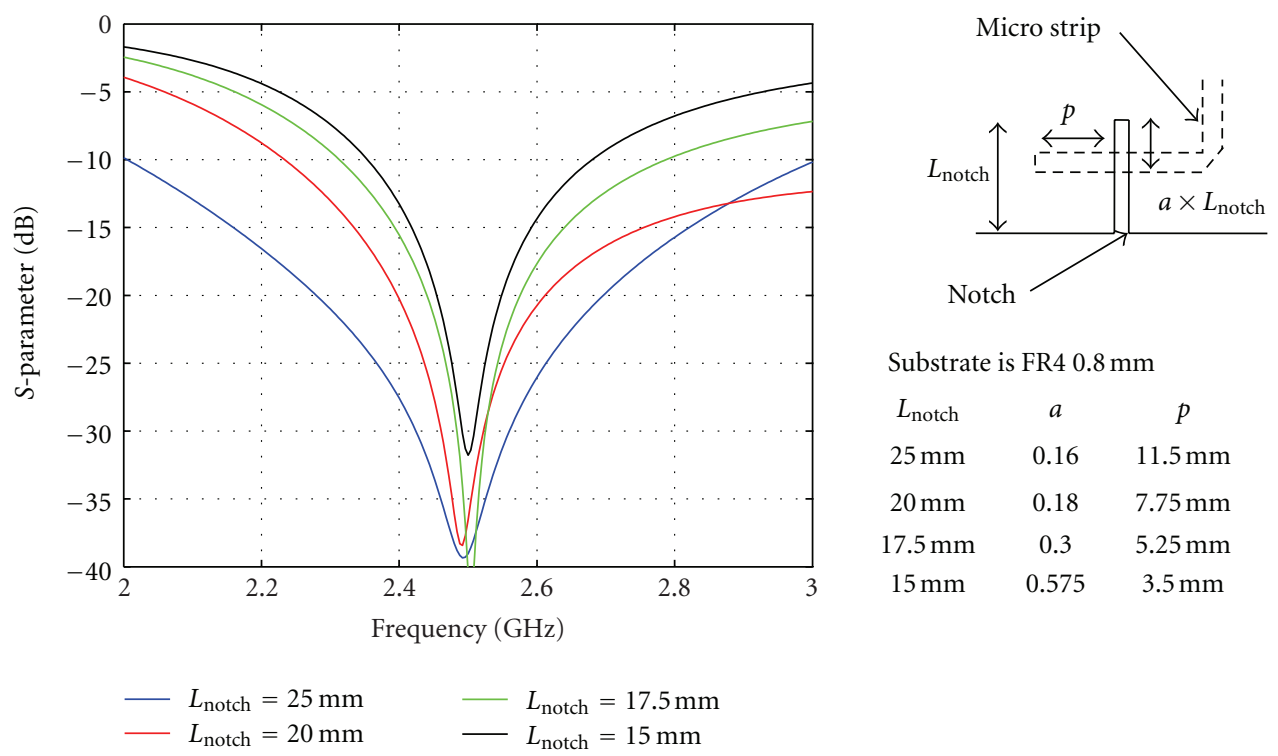

Substrate is FR $40.8 \mathrm{~mm}$

$\begin{array}{ccc}L_{\text {notch }} & a & p \\ 25 \mathrm{~mm} & 0.16 & 11.5 \mathrm{~mm} \\ 20 \mathrm{~mm} & 0.18 & 7.75 \mathrm{~mm} \\ 17.5 \mathrm{~mm} & 0.3 & 5.25 \mathrm{~mm} \\ 15 \mathrm{~mm} & 0.575 & 3.5 \mathrm{~mm}\end{array}$

Figure 2: Impedance matching of notch with reduced length.

different notch lengths. The notch length can be reduced from quarter-wavelength $(\sim 25 \mathrm{~mm})$ if coupling strip is closer to open end of the notch and if stub is short. Consequently the notch can be shortened enough to be cut under PIFA with complete overlap according to Figure $1\left(L_{\text {notch }}=\right.$ $15 \mathrm{~mm})$.

Different placements and orientations of PIFA with respect to combination with notch antenna are investigated by means of simulation (CST microwave studio). The highest coupling level in $2.4-2.5 \mathrm{GHz}$ frequency band is presented in Figure 3. We can observe that best isolation is reached when PIFA and notch are in-line perfectly.

For both PIFA and notch, electrical fields are strong close to open end of the resonators. From Figure 4, we can observe electrical fields in $\pi$ plane defined in Figure 1. Orientation of strongest fields is orthogonal from one element to the other. In this configuration notch antenna can be cut in the ground plane above the PIFA without critical coupling. Close combination of PIFA and notch provides compact multiantenna for MIMO or diversity application at terminal.

\section{Compact Multiantenna Prototype}

IEEE 802.16 WiMax terminals are deployed in $3.4-3.8 \mathrm{GHz}$ frequency band with multiple antennas. We design a prototype of PIFA and notch combination for WiMax network access.

3.1. Multiantenna Geometry. Figure 5 shows compact multiantenna geometry. PIFA has dual resonant lengths which have been jointly optimized in order to widen its operating frequency range [5]. The excitation is performed by means of a metallic post. The PIFA has naturally high radiation efficiency since copper is a good conductor and air substrate is used between radiator and ground plane. The notch is etched in ground plane of a compact printed circuit board (PCB) $25 \mathrm{~mm} \times 35 \mathrm{~mm}$. Radiation efficiency of notch antenna can be significantly reduced by the dielectric losses of the substrate. Therefore, a high performance substrate rogers TMM4 $\left(\tan \delta=2_{E}-3\right)$ has been used and the notch has been partly hollowed from the substrate material close to 


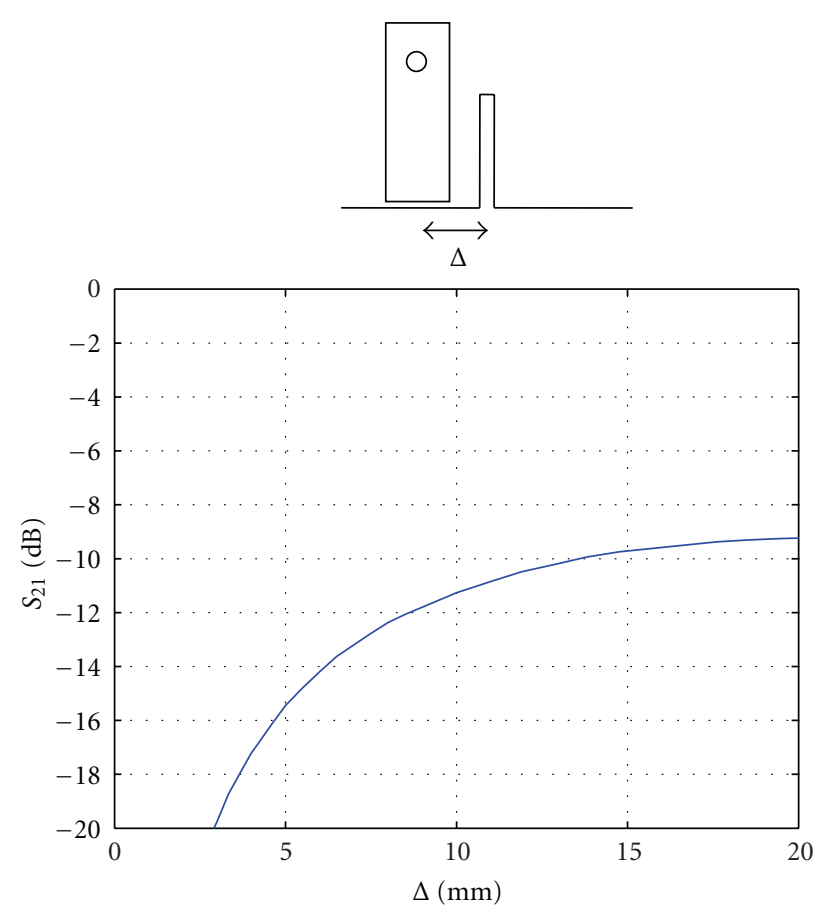

(a)
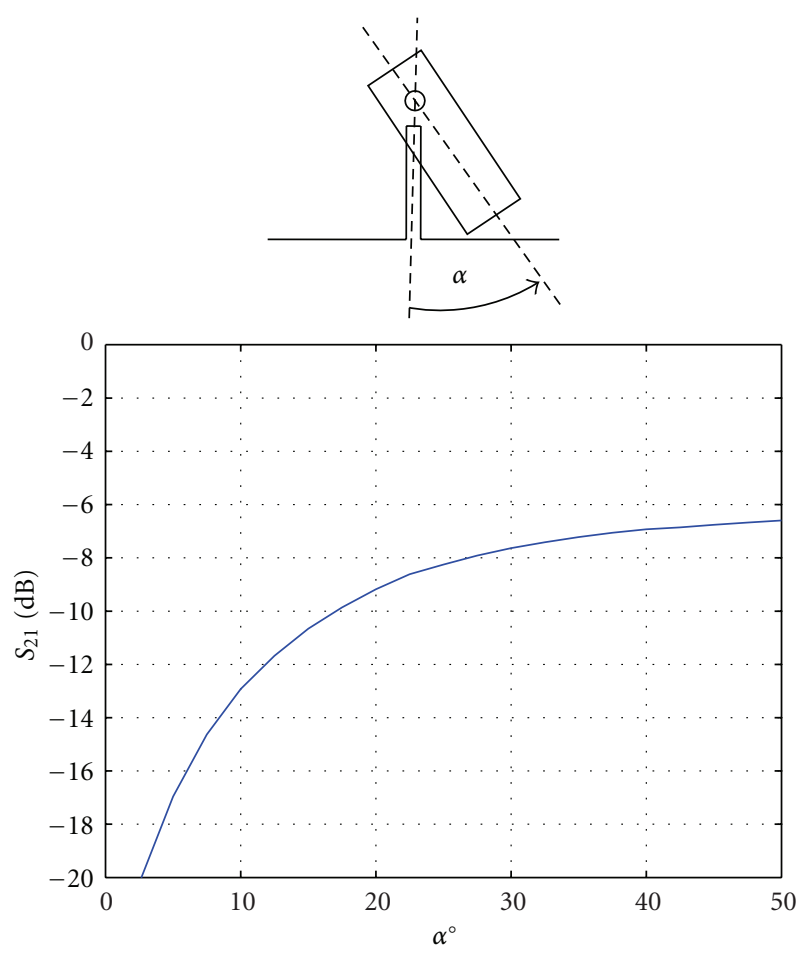

(b)

Figure 3: Investigation of misplacement or rotation of PIFA.

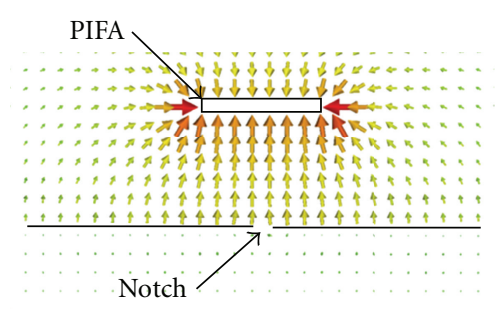

Excitation of PIFA

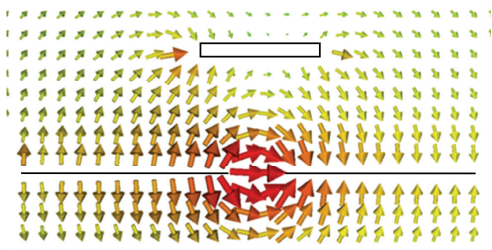

Excitation of notch

FIgURE 4: Electrical fields in open end ( $\pi$ plane).

open end, where resonant electrical fields are strong. Notch antenna is fed by coupling with a micro-strip line etched on the opposite side of the substrate. Impedance matching is reached keeping a short notch length so that it can be located under PIFA with complete overlap. Finally, main dimensions of the multiantenna system are $19 \mathrm{~mm} \times 10 \mathrm{~mm} \times 8 \mathrm{~mm}$, that is, $0.22 \lambda \times 0.11 \lambda \times 0.09 \lambda$, where $\lambda$ is the wavelength in free space at $3.4 \mathrm{GHz}$. Two SMA edge card connectors are mounted on PCB for multiantenna characterization.
3.2. Impedance Matching and Coupling. The scattering parameters of multiantenna prototype are presented in Figure 6. Measurement and CST microwave studio simulation results are, respectively, presented with and without star markers. Ports 1 and 2 are PIFA and notch antenna, respectively. A low reflection coefficient is simultaneously reached at the two ports $3.4-3.6 \mathrm{GHz}$ frequency band. The coupling between the two combined antennas in this bandwidth is low $\left|S_{i j}\right|^{2}<$ $-20 \mathrm{~dB}$. We obtain a quite good agreement between simulation and measurement; slight differences can be explained with respect to the measurement coaxial cable effect.

3.3. Radiation Performances. Gain patterns of the multiantenna prototype at $3.6 \mathrm{GHz}$ are presented in Figure 7 for three orthogonal cut planes. The reference coordinate system is presented in Figure 7 as well. Measurement and CST microwave studio simulation results are, respectively, presented with and without star markers.

We can clearly observe a polarization duality between the two combined antennas in the three considered cut planes: vertically polarized radiation patterns of the PIFA antenna are like the cross-polarized radiation patterns of the notch antenna and vice versa. Each of the two antennas has multiple polarization properties and an omni directional radiation pattern if we consider the elevation cut planes: the multiantenna system is suitable for different environments and/or orientations [4]. Simulation and measurement are in good agreement. We can observe some slight perturbations of the radiation patterns due to the measurement coaxial cables but polarization duality and maximum gain in each cut plane are not significantly affected. 


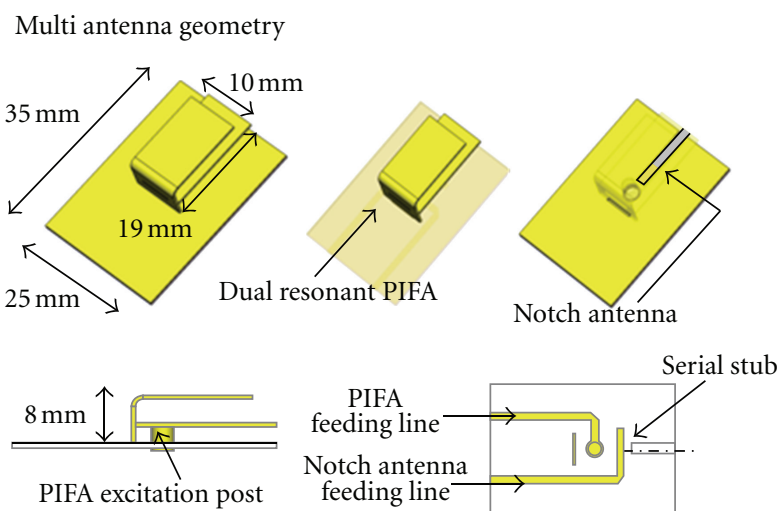

(a)

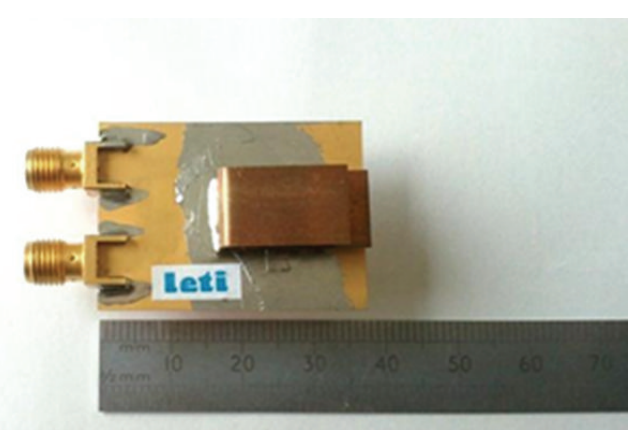

(b)

Figure 5: Compact multiantenna for WiMax.

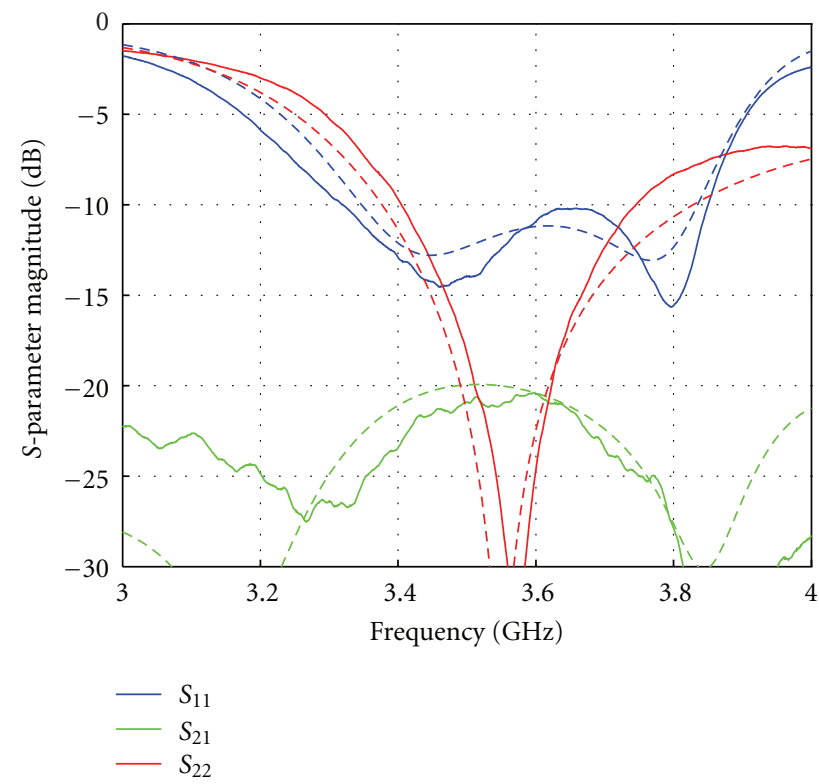

FIGURE 6: Scattering parameters of compact multiantenna prototype. Solid line is measurement, dashed is simulation.

Antenna diversity and MIMO schemes take advantage of uncorrelated radiation properties. The envelop correlation coefficient $\rho$ is computed by means of (1) [6]. If we assume an isotropic environment:

$$
\rho=\frac{\iint E_{1} \cdot E_{2}^{*} d \Omega}{\sqrt{\iint E_{1} \cdot E_{1}^{*} d \Omega \cdot \iint E_{2} \cdot E_{2}^{*} d \Omega}},
$$

$E_{1}$ and $E_{2}$ are radiated electrical far field of PIFA and notch antenna, respectively. $\Omega$ is solid angle unit and ${ }^{*}$ denotes complex conjugate.

Table 1 presents computation results of performance figures based on simulation and measurement results. The radiation efficiency of the PIFA $\left(\eta_{1}\right)$ is high in the $3.4-3.8 \mathrm{GHz}$ frequency band. Also the radiation efficiency of the notch antenna $\left(\eta_{2}\right)$ is high thanks to the poor losses in the rogers TMM4 substrate and the hollowed portion of the substrate in the slot. The multiantenna system provides two signals with
TABLE 1: Multiantenna performances.

\begin{tabular}{lcccccc}
\hline \multirow{2}{*}{ Frequency } & \multicolumn{3}{c}{ Simulation } & \multicolumn{3}{c}{ Measurement } \\
& $\eta_{1}$ & $\eta_{2}$ & $|\rho|$ & $\eta_{1}$ & $\eta_{2}$ & $|\rho|$ \\
\hline $3.4 \mathrm{GHz}$ & 0.97 & 0.96 & 0.04 & 0.94 & 0.97 & 0.05 \\
$3.6 \mathrm{GHz}$ & 0.98 & 0.96 & 0.03 & 0.94 & 0.97 & 0.03 \\
\hline
\end{tabular}

high diversity benefit since the radiation efficiencies are balanced $\eta_{1} \cong \eta_{2}$ and the envelop correlation coefficient is very low $\rho<0.1$ over the targeted frequency band $[6,7]$.

\section{Conclusion}

In this paper, we presented a compact multi antenna. The key concept of this design is heterogeneous combination of antennas. An electric-like PIFA and a magnetic-like notch can 

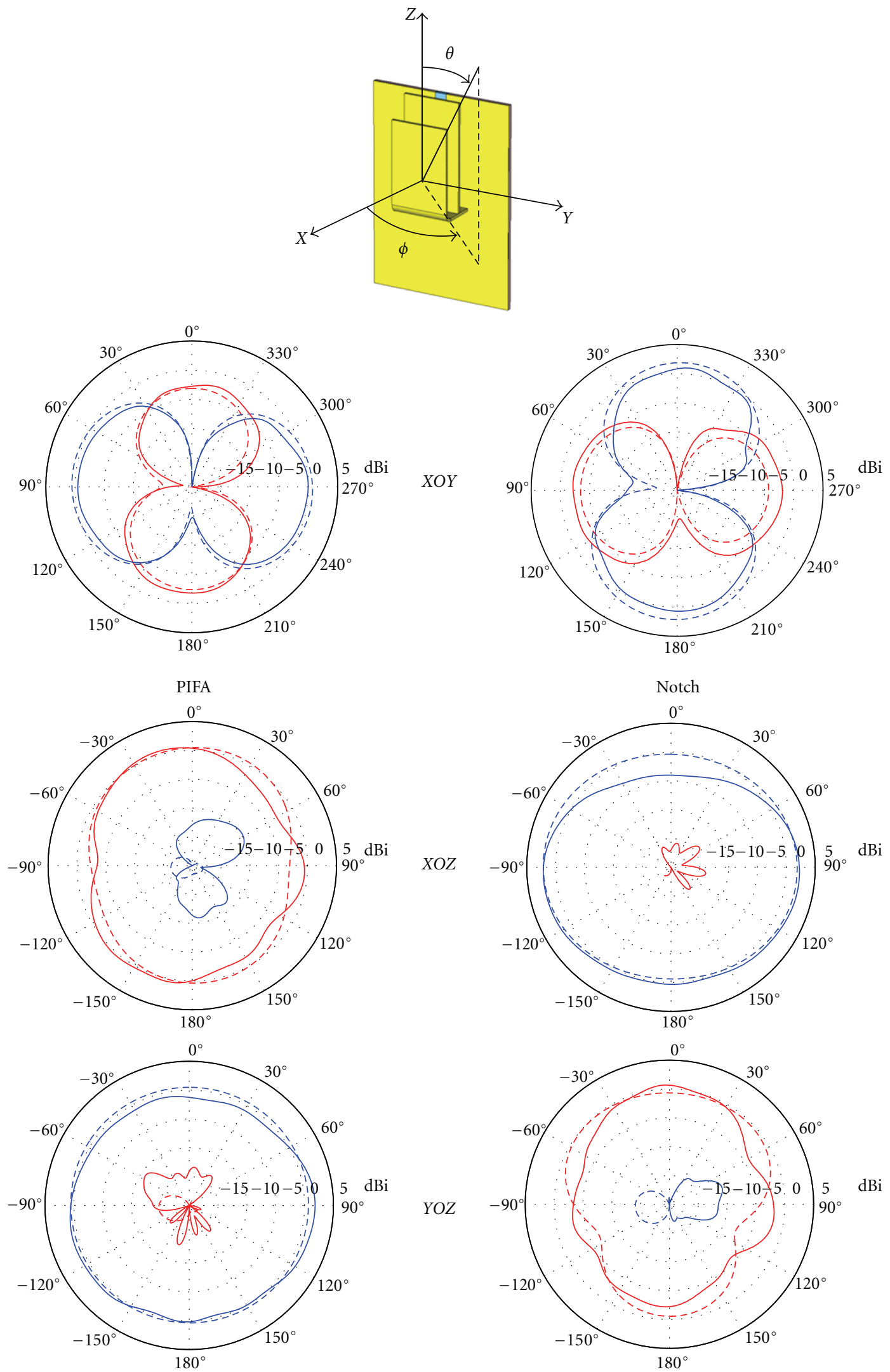

FIGURE 7: Gain patterns of compact multiantenna prototype at $3.6 \mathrm{GHz}$. Solid line is measurement, dashed is simulation. Vertical polarization is red line, horizontal is blue line. 
be colocated in a single volume without strong coupling despite complete overlap. The properties of far-field radiation resulting from duality of combined radiation sources lead to polarization and pattern diversity providing signals weakly correlated. Wideband behavior of this compact antenna system has been demonstrated by designing a prototype optimized for broadband network access in WiMax 3.4-3.8 GHz frequency band. Compact multiantenna system fits in a $0.22 \lambda \times 0.11 \lambda \times 0.09 \lambda$ volume, where $\lambda$ is the free space wavelength at $3.4 \mathrm{GHz}$.

\section{Acknowledgments}

This work was supported in part by the EU commission in the FP7 ROCKET Project and by the French National Research Agency ANR in the ORIANA Project.

\section{References}

[1] C.-Y. Chiu and R. D. Murch, "Overview of multiple antenna designs for handheld devices and base stations," in Proceedings of the IEEE International Workshop on Antenna Technology (iWAT '11), pp. 74-77, Hong Kong, China, March 2011.

[2] J. B. Yan, C. Y. Chiu, and R. D. Murch, "Handset 4-port MIMO antenna using slit separated PIFA and quarterwave-slot antenna pair," in Proceedings of the IEEE International Symposium on Antennas and Propagation, July 2008.

[3] C. Y. Chiu, J. B. Yan, R. D. Murch, J. X. Yun, and R. G. Vaughan, "Design and implementation of a compact 6-port antenna," IEEE Antennas and Wireless Propagation Letters, vol. 8, Article ID 5159465, pp. 767-770, 2009.

[4] L. Rudant and C. Delaveaud, "Heterogeneous combination for compact integrated diversity multi-antenna system," in Proceedings of the 2nd European Conference on Antennas and Propagation (EuCAP'07), Edinburgh, UK, 2007.

[5] R. B. Waterhouse, J. T. Rowley, and K. H. Joyner, "Stacked shorted patch antenna," Electronics Letters, vol. 34, no. 7, pp. 612614, 1998.

[6] R. Vaughan and J. B. Andersen, Channels Propagation and Antennas for Mobile Communications, vol. 50 of IEE Electromagnetic Waves series, 2003.

[7] K. Fujimoto and J. R. James, Mobile Antenna Systems Handbook, Artech House Publishers, 3rd edition, 2008. 

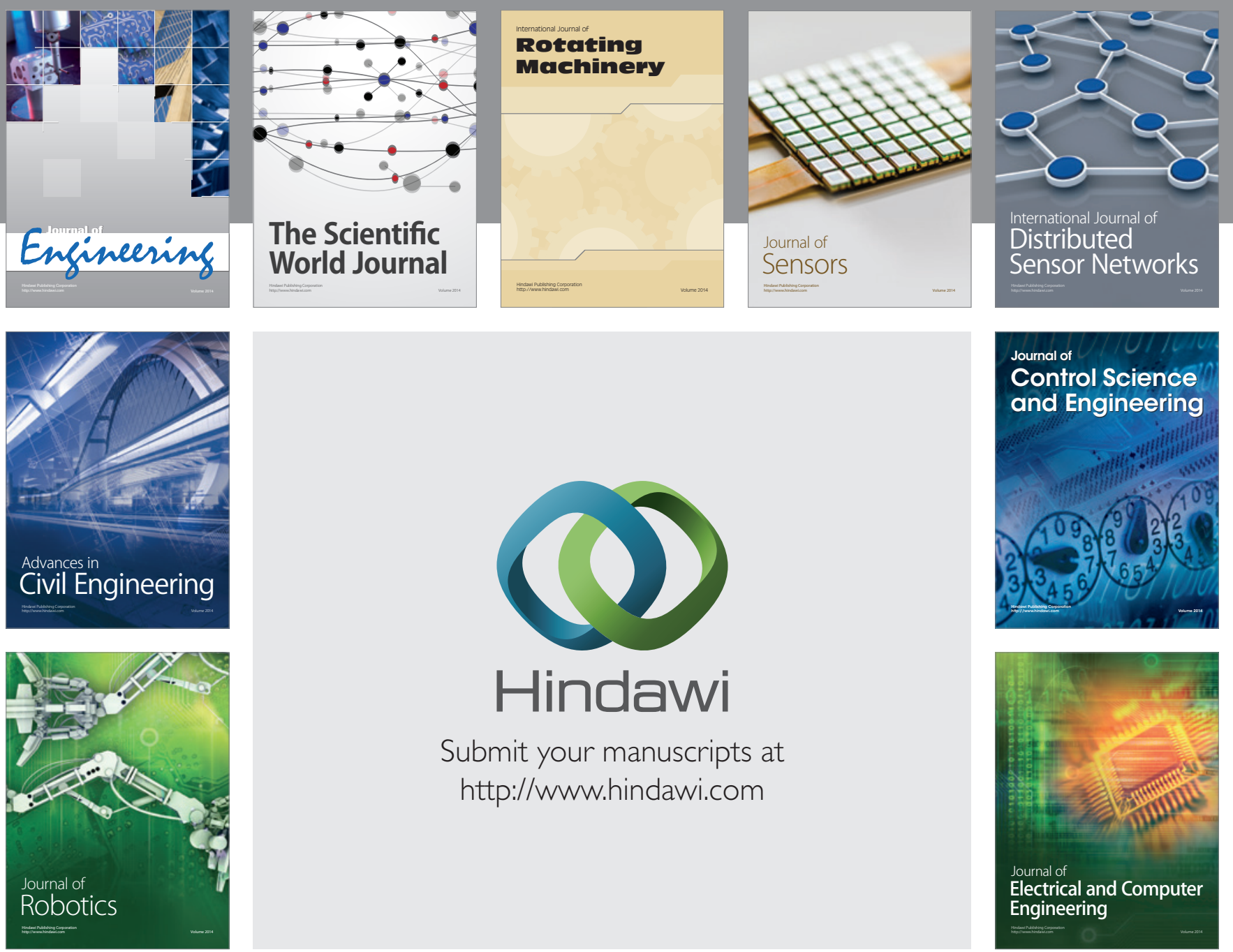

Submit your manuscripts at

http://www.hindawi.com
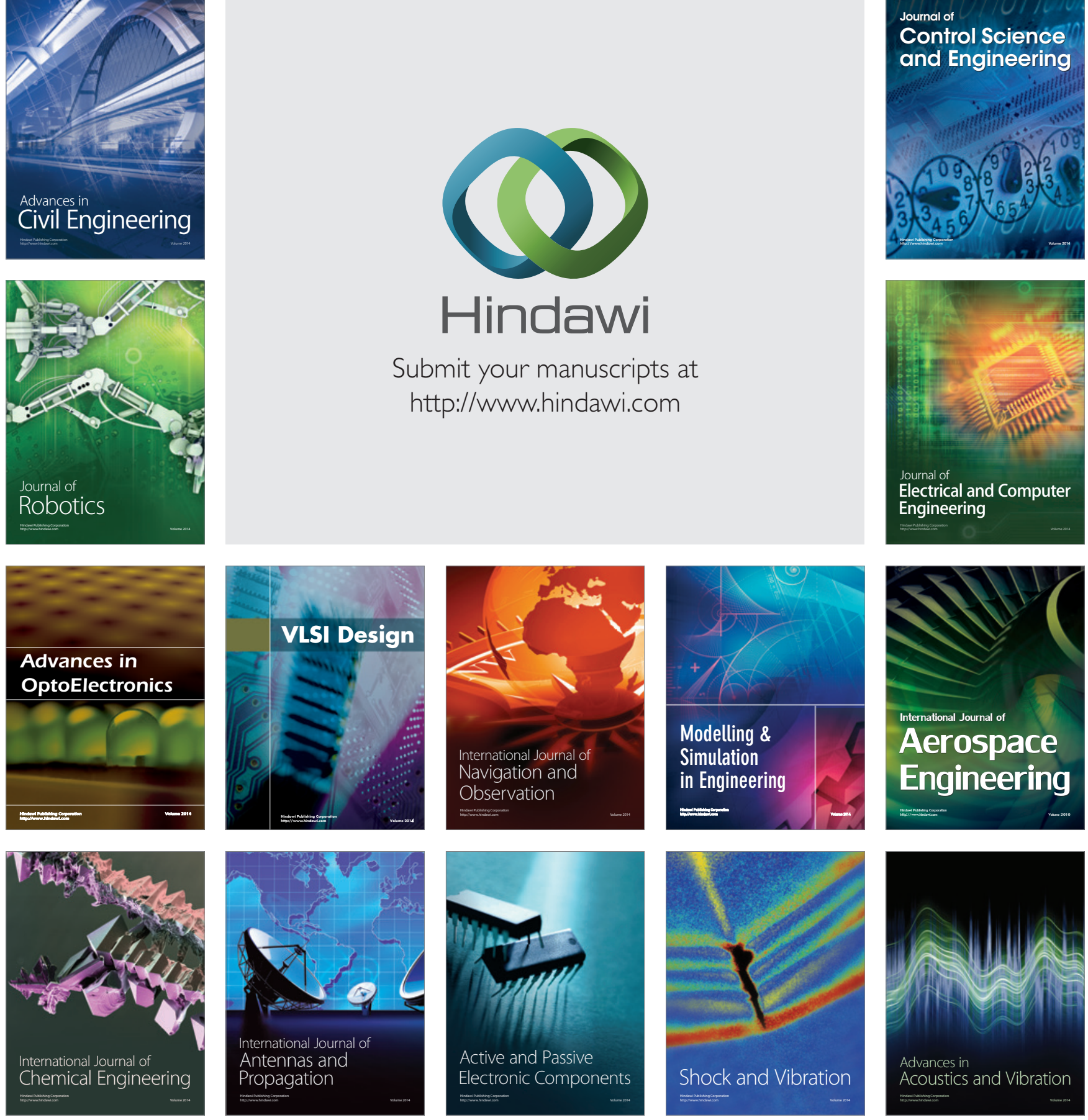